\title{
TOOTH COLOR UNIFORMITY FOLLOWING WHITE SPOT LESION TREATMENT WITH RESIN INFILTRATION OR BLEACHING: IN VITRO STUDY
}

\author{
Heba H. Sawaf, ${ }^{1}$ Hassan E. Kassem, ${ }^{2}$ Nagwa M. Enany ${ }^{3}$
}

\section{ABSTRACT}

Background: Resin infiltration and 6leaching have been used as minimally invasive methods for the treatment of white spot lesions (WSLs) following orthodontic treatment.

Objective: To compare the color uniformity of the tooth with WSL treated with either resin infiltration or bleaching.

Material and Methods: WSLS were created in the right middle buccal half of the enamel surface of 40 sound extracted human premolars. The teeth were randomly assigned to 2 groups $(n=20)$ treated with: (I) resin infiltration, (II) chemically activated officebleaching. Color parameter of the lesion and the adjacent enamel were measured by spectrophotometer at 3 time points: Gaseline (TO), after WSL formation $(\mathcal{T} 1)$, after treatment $(\mathcal{T} 2)$ and color difference $\Delta E^{*}$ was calculated. Each tooth was visually scored for color homogeneity.

Results: $\Delta E^{*}$ was significantly smaller after treatment in group I compared to group II [mean difference $=5.03$ $\pm 1.11, \mathbb{P}<0.0001]$. Group II showed a statistically significant difference after treatment compared to baseline $(P<0.0001)$, whereas group I did not $(\mathcal{P}=$ 1.00). The visual score showed that the tooth color in group I had two-fold chance to be uniform in color compared to group II [Odds ratio $=2.00, \mathbb{P}<0.01$ ]

Conclusions: Treatment of WSLs with resin infiltration result in a more uniform tooth color was achieved compared to 6leaching.
Key words: White spot lesion, office 6leaching, color, resin infiltration, Icon.

\section{INTRODUCTION}

The development of white spot lesions (WSLs) is a common side effect of fixed orthodontic appliance treatment. It has become a serious clinical problem especially in patients with poor oral hygiene. ${ }^{(1)}$ The prevalence of postorthodontic WSLs was reported in $50-75 \%$ of all patients with fixed orthodontic appliances. ${ }^{(2)}$ The severity of WSL may reduce due to nature remineralization; however, stains may get incorporated into the lesion creating brown spots compounding the esthetic challenge. ${ }^{(3)}$ WSLs that fail to remineralize may progress to cavitated carious lesion requiring restorative treatment. ${ }^{(3)}$ In addition, during the remineralization of WSLs, stains can get incorporated into the lesion creating brown spots and thus increasing the esthetic challenge. Hence, the treatment of WSLs attempts to both prevent the caries progression and restore the natural enamel color.

Resin infiltration is a minimally invasive treatment that has been introduced to arrest noncavitated enamel lesions. After erosion of the pseudo-intact surface layer with hydrochloric acid, a low viscosity resin penetrates the lesion filling the porosities of the lesion. Hence, caries progression is slowed down or even arrested. ${ }^{(4)}$ Moreover, the resin infiltrant was found to mask the chalky white appearance of the WSL

(1) Resident, Department of Orthodontics, Faculty of Dentistry, Alexandria University, Alexandria, Egypt.

(1) Lecturer, Department of Orthodontics, Faculty of Dentistry, Alexandria University, Alexandria, Egypt.

(1) Professor, Department of Orthodontics, Faculty of Dentistry, Alexandria University, Alexandria, Egypt. 
approximating the original enamel color. Several studies validated the efficacy of resin infiltration both in vitro ${ }^{(5)}$ and in vivo. ${ }^{(6,7)}$

Post-orthodontic tooth bleaching has grown in popularity in an attempt to remove enamel stains and brighten the enamel color thus enhancing the esthetic result achieved by tooth alignment. ${ }^{(8)}$ The dental bleaching mechanism occurs through an oxidation-reduction chemical reaction where a peroxide reduces the organic pigments impregnated in enamel and dentin eliminating them. ${ }^{(9)}$ Moreover, bleaching has also been used to camouflage post-orthodontic WSL by matching the color of sound enamel to WSL, which was established as a valid option in several studies. $\stackrel{(10-13)}{ }$

Whereas resin infiltration can be applied to the affected teeth confined to the area of the lesion, the bleaching agent is applied to the entire tooth surface and the adjacent teeth to achieve a matching shade. Another important consideration in camouflaging WSLs with bleaching is that the color of WSL will govern the final bleaching shade to be attained to the entire set of teeth; such a shade may be too bright and objectionable to the patient. In addition, the presence of several WSLs with different lightness may pose a conundrum. Several studies compared resin infiltration to bleaching in the treatment of fluorosis $\stackrel{(14,45)}{ }$ and WSLs. ${ }^{(16-18)}$ However, However, there is no direct comparison between the effect of resin infiltration and bleaching on WSLs. Moreover, the results were not always consistent.

Hence, the objective of this study was to evaluate the uniformity of the tooth color affected by a WSL when the lesion was treated with resin infiltration or when it was treated with bleaching.

\section{MATERIALS AND METHODS}

Sample: A sample size of 20 teeth per group was required at $\alpha=0.05$ and a power of $0.80^{(19)}$ to detect a mean difference of $\Delta \mathrm{E}^{*}=3.7^{(20)}$ Forty maxillary premolars extracted for orthodontic purposes were collected from adolescent orthodontic patients requiring maxillary premolar extractions as part of their treatment plan in the Faculty of Dentistry, Alexandria University. Teeth were excluded in case of the presence of environmental or developmental enamel defects such as enamel hypoplasia or fluorosis, decalcifications, restoration, previous bonding or chemical treatment. Immediately following extraction, the teeth were scaled, thoroughly cleansed under running tap water and stored in distilled water at room temperature to avoid dehydration until the time of the experiment.

Grouping: The teeth were randomly assigned to 2 groups $(n=20)$ according to the treatment performed: group I, resin infiltration, and group II, office bleaching. A random number list was generated using Microsoft Excel (Microsoft Corporation, Redmond, WA USA). The allocation was concealed in sealed envelopes until the implementation of the treatment. Moreover, the investigator was blinded to the allocation during outcome assessment.

\section{Intervention:}

Before induction of WSL, 2 layers of acid resistant nail varnish were applied to cover the whole crown and root leaving the right halve of the middle third of the buccal surface of the enamel surface of each specimen unprotected. Artificial enamel subsurface lesions were created in the unprotected area by immersion in a demineralization solution $^{(21)}$ for 4 days. The demineralizing solution consisted of: (2.2 $\mathrm{mmol} / \mathrm{L}$ calcium chloride, $2.2 \mathrm{mmol} / \mathrm{L}$ 
monopotassium phosphate, $50 \mathrm{mmol} / \mathrm{L}$ acetic acid, $1 \mathrm{~mol} / \mathrm{L}$ potassium hydroxide).

Icon resin infiltration (Icon®, DMG, Hamburg, Germany) was applied according to the manufacturer's instructions (Fig.1). The surface layer of the lesions was eroded with $15 \%$ hydrochloric acid gel (Icon-Etch) for 2 min. and then rinsed with water spray for 30 sec., dried with oil and water free air, followed by the application of $99 \%$ ethanol (Icon-Dry) for $30 \mathrm{sec}$., then air dried. Two layers of the low viscosity resin infiltrant (Icon-Infiltrant) were applied to the etched lesion where the first layer was left for $3 \mathrm{~min}$. then light cured for $40 \mathrm{sec}$; and the second layer was applied for 1 min., the excess material was then removed and light cured again for $40 \mathrm{sec}$.

Chemically activated office-bleaching system (Opalescence Boost 40\% Hydrogen Peroxide, Ultradent, South Jordan, UT, USA) was applied as shown in figure 2. The bleaching system consists of 2 syringes: one containing the activator and the other hydrogen peroxide. The 2 components were mixed together according to the manufacturer's instructions. The gel was then applied as a $1-2 \mathrm{~mm}$ thick layer to the entire buccal surface for $20 \mathrm{~min}$. then thoroughly rinsed with water.

Assessment: The color of each tooth half was measured by the principal investigator using the VITA Easyshade spectrophotometer (VITA Zahnfabrik, Bad Säckingen, Germany). Measurements were carried out by the same investigator in the same examination room against a black background in a specialized dark box. The probe tip was held at $90^{\circ}$ to each half of the tooth surface (Fig.3). ${ }^{(22)}$ The color of each tooth half was measured using the spectrophotometer at 3 time points: before treatment at baseline (T0), following WSL formation (T1), following treatment (T2). The colorimetric evaluation was performed according to the CIE L*a*b* order system that defines the color of an object within a 3 dimensional color space using 3 parameters where: $\mathrm{L}^{*}$ corresponds to the value or degree of lightness ( $\mathrm{L}+=$ lightness and $\mathrm{L}-=$ darkness) $\mathrm{a}^{*}$ coordinates designate position on red/green axis $\left(\mathrm{a}^{*}+=\right.$ redness and $\mathrm{a}^{*}$ $=$ greenness) $b^{*}$ coordinates designate position on yellow/blue axis $\left(b^{*}+=\right.$ yellowness and $b^{*}-=$ blueness). Three consecutive readings were measured by the spectrophotometer, the mean values of $\mathrm{L}^{*}, \mathrm{a}^{*}$, and $\mathrm{b}^{*}$ and the difference in $\mathrm{L}^{*}$ $(\Delta \mathrm{L})^{*}, \mathrm{a}^{*}(\Delta \mathrm{a})^{*}$, and $\mathrm{b}^{*}(\Delta \mathrm{b})^{*}$ between the 2 halves of the tooth were calculated. The color difference between the 2 halves $\left(\Delta \mathrm{E}^{*}\right)$ of each tooth was calculated for each tooth according to the following formula ${ }^{(23)}$ :

$\Delta \mathrm{E}^{*}=\left[\left(\Delta \mathrm{L}^{*}\right)^{2}+\left(\Delta \mathrm{a}^{*}\right)^{2}+\left(\Delta \mathrm{b}^{*}\right)^{2}\right] 1 / 2$

\section{Visual score:}

Each tooth was examined visually in the same examination room with standardized lighting conditions (Ceiling mounted 5500 Kelvin LED light) and the natural day light was excluded using an optical opaque louver. ${ }^{(24)}$ During examination, each tooth was placed on the bench against a black background and in a 30 $\mathrm{cm}$ fixed distance. ${ }^{(25)}$ A scoring system was used to assess the homogeneity of the tooth color: 0 for uniform enamel color or clinically accepted or 1 for non-uniform enamel color warranting further intervention.

\section{Statistical Analysis:}

Intra class correlation coefficient (ICC) was used to test the reliability of color difference $\left(\Delta \mathrm{E}^{*}\right)$. ICC was $0.90[95 \% \mathrm{CI}=(0.872,0.933)]$ and $0.85[95 \% \mathrm{CI}=(0.740,0.918)]$ for intrarater and inter-rater reliability, respectively indicating excellent agreement. Normality was confirmed with histograms and Shapiro Wilk 
test. $\Delta \mathrm{E}^{*}$ was presented using means and standard deviation. Repeated measures ANOVA was used to test for the compare the interventions at the different time points. Post hoc multiple comparisons were done by
Bonferroni tests. One sample t-test was used to compare $\Delta \mathrm{E} *$ to the perceptibility threshold value 3.7. Visual score was presented as percentages and odds ratio of color uniformity was calculated.
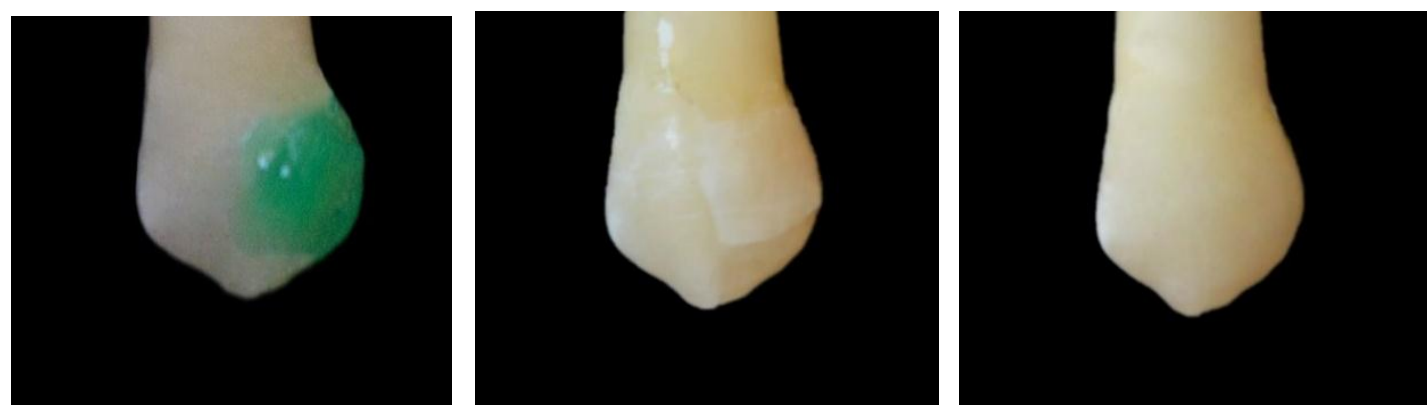

Fig (1): Steps of Icon application, A: Icon-Etch; B: Icon-Dry; C: Icon-Infiltrant.
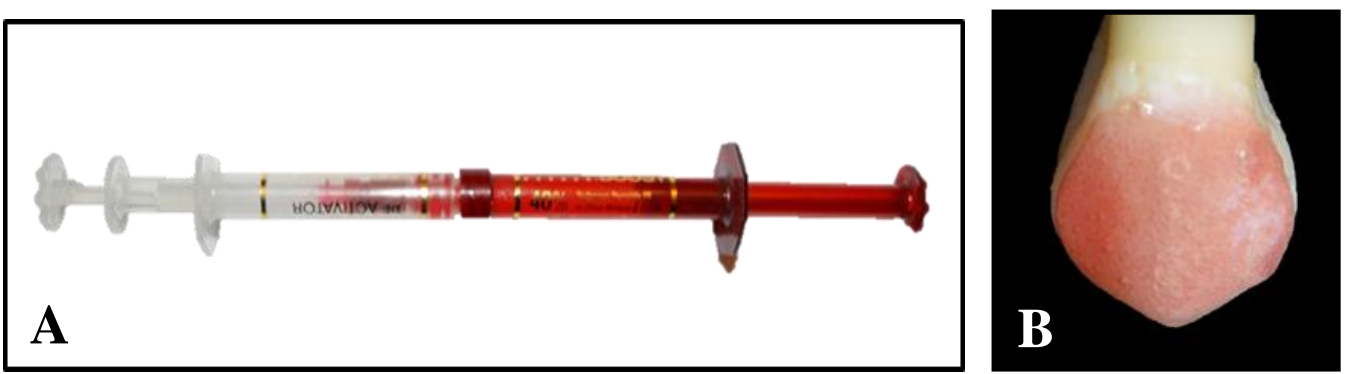

Fig (2): A: Opalescence Xtra Boost B: Application of bleaching agent applied on the entire buccal tooth surface.
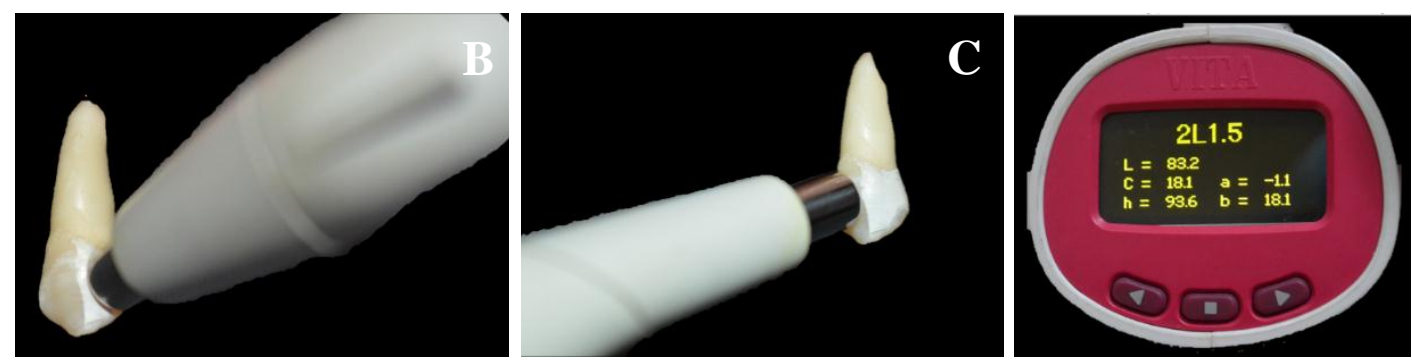

Fig (3): A: spectrophotometer measuring right buccal suface of each specimen on which the WSL was formed B: spectrophotometer measuring left buccal surface of each specimen. C: the analysis displayed on the spectrophotometer screen giving the components of color $\mathrm{L}^{*}, \mathrm{a}^{*}$, and $\mathrm{b}^{*}$. 


\section{RESULTS}

There was a statistically significant increase in $\Delta \mathrm{E}^{*}$ following the induction of WSL at T1 in both group I $[8.41 \pm 1.72,95 \% \mathrm{CI}(3.61,13.21)$ and $\left.\mathrm{P}<0.0001^{*}\right]$ as well as in group II [10.31 $\pm 1.72,95 \%$ CI $(5.51,15.11)$ and $\mathrm{P}<0.0001 *]$. Both groups showed a statistically significant decrease in $\Delta E^{*}$ following resin infiltration in group I $[-8.18 \pm 1.41,95 \%$ CI $(-12.13,-4.24)$ and $\left.\mathrm{P}=0.0001^{*}\right]$ and bleaching in group II [$4.15 \pm 1.41,95 \% \mathrm{CI}(-8.10,0.21)$ and $\mathrm{P}=$ 0.03*] compared to T1. Compared to baseline (T0), $\Delta \mathrm{E}^{*}$ showed no statistically significant difference in group I $[0.22 \pm 0.93,95 \%$ CI ($2.37,2.82$ ) and $\mathrm{P}=1.00]$. However, in group II, there was a statistically significant difference between $\mathrm{T} 2$ and $\mathrm{T} 0[6.16 \pm 0.93$, 95\% CI $(3.56,8.76)$ and $\mathrm{P}=0.0001^{*}$. (Table 1, Fig. 4)

$90 \%$ of the specimens scored 0 in group I compared to $50 \%$ in group II. The odds ratio showed that group I has two-fold chance of color uniformity compared to group II [Odds ratio $=2.00 ; 95 \%$ CI $1.27,31.4, \mathrm{P}<0.01)$. (Figs. 4, 5)

Table 1: Mean $\Delta E^{*}$ of group I and group II at different time points

\begin{tabular}{|c|c|c|}
\hline & $\begin{array}{c}\text { Group I } \\
\text { Resin Infiltration } \\
(n=20)\end{array}$ & $\begin{array}{c}\text { Group II } \\
\text { Bleaching } \\
(n=20)\end{array}$ \\
\hline & \multicolumn{2}{|c|}{$\operatorname{Mean}(S D) *$} \\
\hline TO & $7.27(3.99)^{\mathrm{A}, \mathrm{a}}$ & $6.37(2.78)^{\mathrm{A}, \mathrm{a}}$ \\
\hline$T 1$ & $15.68(6.14)^{\mathrm{A}, \mathrm{b}}$ & $16.68(7.36)^{\mathrm{A}, \mathrm{b}}$ \\
\hline$T 2$ & $7.49(2.74)^{\mathrm{A}, \mathrm{a}}$ & $12.52(4.18)^{\mathrm{B}, \mathrm{c}}$ \\
\hline
\end{tabular}

* Based on significant repeated measures ANOVA.Post hoc comparison testing Bonferroni multiple tests. Different capital letters (A, B) indicate statistically significant difference across rows;

different small letters $(\mathrm{a}, \mathrm{b}, \mathrm{c})$ indicate statistically significant difference across columns. $\boldsymbol{P} \leq 0.05$

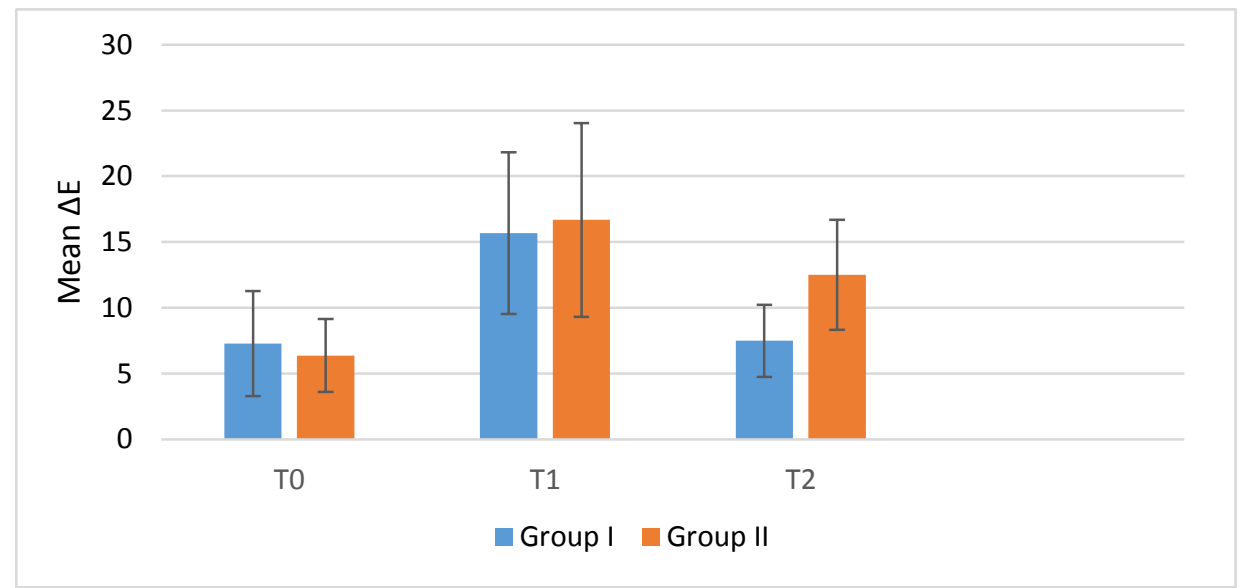

Fig (4): Mean $\Delta \mathrm{E}^{*}$ with in group I and group II at different time points. Error bars represent S.D. 

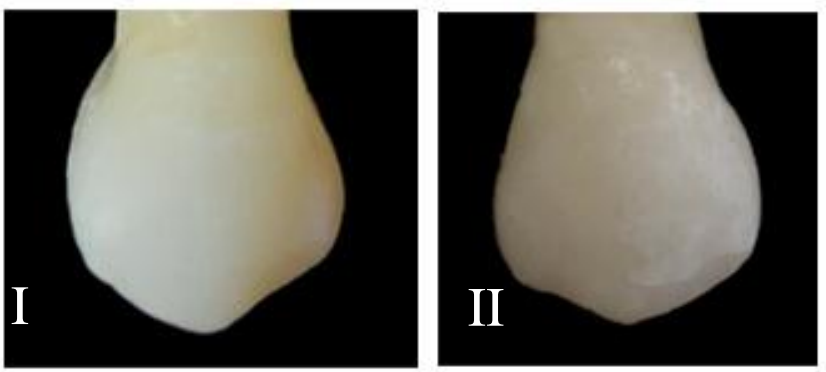

Fig (5): Representative photographs comparing tooth color after treatment. I, II: Group I and group II, respectively

\section{DISCUSSION}

One of the prime objectives of orthodontic treatment is the preservation of the health of oral tissues. WSLs may impair the esthetic appearance of the tooth enamel, thus decreasing the satisfaction with the outcome of an otherwise successful orthodontic therapy.(26) In addition, WSLs carry the risk of progression into a cavitated carious lesion increasing treatment burden.

Although in vitro studies cannot fully simulate the physiologic conditions of the oral cavity, however, it allows for the comparison of different materials under standardized conditions. Artificially induced WSLs were used to study the remineralization of enamel owing to their histological similarity to natural WSLs. $(27,28)$ In the current study, the WSLs were induced by immersing the premolars in a demineralizing solution at acidic $\mathrm{pH} 4.5$ for 96 hours. This duration was chosen because it was found to produce visible WSLs without cavitation. The presence of calcium and phosphate ions in the demineralizing solution served to decrease the loss of the tooth surface.(29)

In the present study, the uniformity of the color was assessed by comparing one half of the tooth to the other and the difference $\Delta \mathrm{E}^{*}$ was calculated between them. This study design serves the purpose of study color uniformity. In addition, this avoid the calculation of $\Delta \mathrm{E}^{*}$ between time points or between different groups which might render statistical comparisons difficult. Moreover, it might reduce the difficulty of measurement on the convex buccal surface of maxillary premolars in case the WSL was created in the center of the buccal surface. This study design was used in several studies.(30-33)

The Vita Easyshade spectrophotometer was shown to be the most accurate instrument compared to Spectroshade spectrophotometer, as well as Shade Vision, Shade Scan and color measurements using digital photographs.(34) Several measures were taken to ensure consistent color measurements. The spectrophotometer was calibrated at the beginning of every color measurement session. The investigator was trained to ensure reliability of measurement and area of measurement before outcome assessment. Three measurements were taken and the average of the closest two measurements was computed. In addition, all the measurements were taken in the same room under standardized lighting and against a uniform background. Similar precautions were undertaken during visual scoring. $(24,25)$

According to Lim et al.,(35) visual color determination is considered to be subjective, compared to the accuracy of spectrophotometric measurements. However, Vachon et al.(36) suggested that although the spectrophotometer readings might indicate a statistically significant difference, these differences could be clinically identical to the human eye. Therefore, some studies have used 
both the spectrophotometer and visual scoring to evaluate the effectiveness of resin infiltration and bleaching agents in color difference. The results of both methods were found to be consistent. $(37,38)$

In the present study, the difference in color between the WSL and the sound adjacent enamel in group I after the application of Icon has decreased near to baseline value. The efficacy of resin infiltration in treating WSLs has already been demonstrated in many studies.(39)

An improvement in the color difference between the WSL and adjacent bleached enamel in group II was seen after bleaching compared to the color difference after decalcification. Bleaching was demonstrated to be effective in the treatment of WSLs in many studies.(10-13)

In this study, a more uniform enamel color was found with resin infiltration (group I) compared to bleaching (group II). This has been demonstrated in a lower color difference following treatment at $\mathrm{T} 2$ in group I. In addition, there was no statistically significant difference between group I at T2 compared to baseline at $\mathrm{T} 1$. The visual score corroborated the spectrophotometric findings where group II was twice more likely to have a uniform enamel color compared to group. It is worth pointing out that $\Delta \mathrm{E}^{*}$ after treatment at $\mathrm{T} 2$ in group I was calculated between the color parameter of a resin-infiltrated WSL and the adjacent untreated enamel. However, in group II, the two halves of the tooth were bleached WSL versus bleached enamel.

The results of this study are consistent with a study conducted by Gugnani et al.(14) who evaluated the effect of resin infiltration, bleaching and their combination in non-pitted fluorosis stains. The visual analogue scale showed that the best results were found in the group treated with resin infiltration with double application of the resin infiltrant followed by the resin infiltrated group then the bleached and resin infiltrated group and the least improvement was in the bleached group.
Similarly, Schoppmeier et al.(15) found greater reduction in color difference relative to the baseline following resin infiltration compared to light activated office bleaching in the treatment of enamel fluorosis. However a direct statistical comparison was not reported.

On the other hand, Torres et al.(18) demonstrated that resin infiltration increased enamel fluorescence immediately enamel whereas bleaching reduced the fluorescence to its baseline values. Similarly, Araujo et al.(16) reported a larger $\mathrm{L}^{*}$ value was reported upon bleaching of stained WSL compared to nonstained WSL treated with resin infiltration, however no direct statistical comparison was reported. Nevertheless, it can be argued that no direct comparison was made with the surrounding enamel. Moreover, differences in fluorescence may not be directly extrapolated to changes in color. On the contrary, Horuzetepe et al.(17) found no statistically significant difference between resin infiltration and office bleaching comparing the color difference after treatment to baseline.

It can be argued that a single application of bleaching may not be sufficient to camouflage the color of the WSL. However, the objective of this study was not to test the efficacy of bleaching in masking the WSL per se. It has been demonstrated that a conservative application of the bleaching agent might not efficacious, hence multiple applications of the bleaching agents may be required resulting in too bright a shade that will be objectionable to the patient.

The findings of this study should be viewed in the light of the transferability of the in vitro study design results to clinical situations, the inherent errors of spectrophotometric measurements $(40,41)$ and the lack of a long term follow-up of the color stability under $\mathrm{pH}$ cycling.(17)

\section{CONCLUSION}

Based on the results of the current study, resin infiltration of WSL results in a more uniform enamel color compared to office-bleaching. 


\section{REFERENCES}

1. Øgaard B. White Spot Lesions During Orthodontic Treatment: Mechanisms and Fluoride Preventive Aspects. Semin Orthod. 2008;14(3):183-93.

2. Gorelick L, Geiger AM, Gwinnett AJ. Incidence of white spot formation after bonding and banding. Am J Orthod. 1982;81(2):93-8.

3. Kidd EAM, Fejerskov O. What Constitutes Dental Caries? Histopathology of Carious Enamel and Dentin Related to the Action of Cariogenic Biofilms. J Dent Res. 2016;83(1_suppl):35-8.

4. Paris S, Meyer-Lueckel H, Kielbassa A. Resin infiltration of natural caries lesions. J Dent Res. 2007;86(7):662-6.

5. Paris S, Meyer-Lueckel H. Inhibition of caries progression by resin infiltration in situ. Caries Res. 2010;44(1):47-54.

6. Paris S, Hopfenmuller W, MeyerLueckel H. Resin infiltration of caries lesions: an efficacy randomized trial. J Dent Res. 2010;89(8):823-6.

7. Ekstrand KR, Bakhshandeh A, Martignon S. Treatment of proximal superficial caries lesions on primary molar teeth with resin infiltration and fluoride varnish versus fluoride varnish only: efficacy after 1 year. Caries Res. 2010;44(1):41-6.

8. Ghanbarzadeh M, Ahrari F, Akbari M, Hamzei H. Microhardness of demineralized enamel following home bleaching and laserassisted in office bleaching. J Clin Exp Dent. 2015;7(3):e405-9.

9. Lunardi N, Correr AB, Rastelli AN, Lima DA, Consani RL. Spectrophotometric evaluation of dental bleaching under orthodontic bracket in enamel and dentin. J Clin Exp Dent. 2014;6(4):e321-6.

10. Knosel M, Attin R, Becker K, Attin T. External bleaching effect on the color and luminosity of inactive white-spot lesions after fixed orthodontic appliances. Angle Orthod. 2007;77(4):646-52.
11. Kim Y, Son HH, Yi K, Ahn JS, Chang J. Bleaching effects on color, chemical, and mechanical properties of white spot lesions. Oper Dent. 2016;41(3):318-26.

12. Ahrari F, Akbari M, Mohammadpour S, Forghani M. The efficacy of laser-assisted inoffice bleaching and home bleaching on sound and demineralized enamel. Laser Ther. 2015;24(4):257-64.

13. Choi YY, Lee DY, Kim YJ. Colorimetric evaluation of white spot lesions following external bleaching with fluoridation: An in-vitro study. Korean J Orthod. 2018;48(6):377-83.

14. Gugnani N, Pandit IK, Gupta M, Gugnani S, Soni S, Goyal V. Comparative evaluation of esthetic changes in nonpitted fluorosis stains when treated with resin infiltration, in-office bleaching, and combination therapies. J Esthet Restor Dent. 2017;29(5):317-24.

15. Schoppmeier CM, Derman SHM, Noack MJ, Wicht MJ. Power bleaching enhances resin infiltration masking effect of dental fluorosis. A randomized clinical trial. J Dent. 2018;79:77-84.

16. Araujo GS, Naufel FS, Alonso RC, Lima DA, Puppin-Rontani RM. Influence of Staining Solution and Bleaching on Color Stability of Resin Used for Caries Infiltration. Oper Dent. 2015;40(6):E250-6.

17. Horuztepe SA, Baseren M. Effect of resin infiltration on the color and microhardness of bleached white-spot lesions in bovine enamel (an in vitro study). J Esthet Restor Dent. 2017;29(5):378-85.

18. Torres CRG, Zanatta RF, Fonseca BMd, Borges AB. Fluorescence properties of demineralized enamel after resin infiltration and dental bleaching. AM J DENT. 2019;32(1):43-6.

19. Daniel WW. A foundation for analysis in the health sciences. Biostatistics. 1995:5918.

20. Johnston WM, Kao EC. Assessment of appearance match by visual observation and 
clinical colorimetry. J Dent Res. 1989;68(5):819-22.

21. ten Cate JM, Duijsters PP. Alternating demineralization and remineralization of artificial enamel lesions. Caries Res. 1982;16(3):201-10.

22. AlGhazali N, Burnside G, Smith P, Preston A, Jarad F. Performance assessment of Vita Easy Shade spectrophotometer on colour measurement of aesthetic dental materials. European Journal of Prosthodontics and Restorative Dentistry. 2011;19(4):168.

23. Comission Internationale de l'Eclairage. (1977). CIE Recommendations on uniform color spaces, color-difference equations, and metric color terms.

24. Lehmann KM, Igiel C, Schmidtmann I, Scheller H. Four color-measuring devices compared with a spectrophotometric reference system. J Dent. 2010;38 Suppl 2:e65-70.

25. Basavanna RS, Gohil C, Shivanna V. Shade selection. International Journal of Oral Health Sciences. 2013;3(1):26.

26. Ellwood RP, O'Mullane D. Enamel opacities and dental esthetics. Journal of Public Health Dentistry. 1995;55(3):171-6.

27. Coolidge TB, Besic FC, Jacobs MH. A microscopic comparison of clinically and artificially produced changes in enamel. Oral surgery, oral medicine, and oral pathology. 1955;8(11):1204-10.

28. Soni NN, Brudevold F. Microradiographic and polarized-light studies of artificially produced lesions. J Dent Res. 1960;39(2):233-40.

29. Jeong $\mathrm{S}$, Jang $\mathrm{S}$, Kim $\mathrm{KN}$, Kwon $\mathrm{H}$, Park YD, Kim B, editors. Remineralization potential of new toothpaste containing nanohydroxyapatite. Key Engineering Materials; 2006: Trans Tech Publ.

30. Hallgren K, Akyalcin S, English J, Tufekci E, Paravina RD. Color Properties of Demineralized Enamel Surfaces Treated with a Resin Infiltration System. J Esthet Restor Dent. 2016;28(5):339-46.
31. Zhao X, Ren YF. Surface Properties and Color Stability of Resin-Infiltrated Enamel Lesions. Oper Dent. 2016;41(6):617-26.

32. Leland A, Akyalcin S, English JD, Tufekci E, Paravina R. Evaluation of staining and color changes of a resin infiltration system. Angle Orthod. 2016;86(6):900-4.

33. Mazur M, Westland S, Guerra F, Corridore D, Vichi M, Maruotti A, et al. Objective and subjective aesthetic performance of icon( $R$ ) treatment for enamel hypomineralization lesions in young adolescents: A retrospective single center study. J Dent. 2018;68:104-8.

34. Kim-Pusateri S, Brewer JD, Davis EL, Wee AG. Reliability and accuracy of four dental shade-matching devices. J Prosthet Dent. 2009;101(3):193-9.

35. Lim MY, Lum SO, Poh RS, Lee GP, Lim KC. An in vitro comparison of the bleaching efficacy of $35 \%$ carbamide peroxide with established intracoronal bleaching agents. International endodontic journal. 2004;37(7):483-8.

36. Vachon C, Vanek P, Friedman S. Internal bleaching with $10 \%$ carbamide peroxide in vitro. 1998.

37. Nathoo S, Zhang Y, Lin N, Collins M, Klimpel K, DeVizio W, et al. Comparative seven-day clinical evaluation of two tooth whitening products. Compend Contin Educ Dent. 2001;22(7):599-604, 6; quiz 8.

38. Li Y, Lee SS, Cartwright SL, Wilson AC. Comparison of clinical efficacy and safety of three professional at-home tooth whitening systems. Compend Contin Educ Dent. 2003;24(5):357-60, 62, 64 passim; quiz 78.

39. Borges AB, Caneppele TM, Masterson $\mathrm{D}$, Maia LC. Is resin infiltration an effective esthetic treatment for enamel development defects and white spot lesions? A systematic review. J Dent. 2017;56:11-8.

40. Berns RS, Petersen KH. Empirical modeling of systematic spectrophotometric errors. Color Research \& Application. 1988;13(4):243-56. 
41. Ten Bosch J, Coops J. Tooth color and reflectance as related to light scattering and enamel hardness. J Dent Res. 1995;74(1):37480. 